\title{
Impact of COVID-19 on Multiple Sclerosis Topic Discussion on Twitter
}

\author{
Guido GIUNTI a,b,1 , Maëlick CLAES ${ }^{\mathrm{a}}$, Enrique Dorronzoro ZUBIETE ${ }^{\mathrm{c}}$, Octavio \\ RIVERA-ROMERO ${ }^{\mathrm{c}, \mathrm{d}}$ and Elia GABARRON ${ }^{\mathrm{e}}$ \\ a University of Oulu, Finland \\ ${ }^{b}$ TU Delft, The Netherlands \\ ${ }^{c}$ Universidad de Sevilla, Spain \\ ${ }^{d}$ I3US: Research Institute of Computer Engineering, Universidad de Sevilla, Spain \\ ${ }^{e}$ Norwegian Centre for E-health Research, University Hospital North Norway, Norway
}

\begin{abstract}
Introduction: Multiple sclerosis (MS) is one of the world's most common neurologic disorders. Social media have been proposed as a way to maintain and even increase social interaction for people with MS. The objective of this work is to identify and compare the topics on Twitter during the first wave of COVID-19 pandemic. Methods: Data was collected using the Twitter API between 9/2/2019 and $13 / 5 / 2020$. SentiStrength was used to analyze data with the day that the pandemic was declared used as a turning point. Frequency-inverse document frequency (tf-idf) was used for each unigram and calculated the gains in tf-idf value. A comparative analysis of the relevance of words and categories among the datasets was performed. Results: The original dataset contained over 610k tweets, our final dataset had 147,963 tweets. After the 10th of march some categories gained relevance in positive tweets ("Healthcare professional", "Chronic conditions", "Condition burden"), while in negative tweets "Emotional aspects" became more relevant and "COVID-19" emerged as a new topic. Conclusions: Our work provides insight on how COVID-19 has changed the online discourse of people with MS.
\end{abstract}

Keywords. Multiple Sclerosis; COVID-19; Social Media; Sentiment Analysis

\section{Introduction}

Multiple sclerosis (MS) is one of the most common progressive neurologic disorders in young adults [1]. Social support is an important aspect for people with MS (pwMS) [2]. Maintaining social activities improves the quality of life for pwMS [3]. Social media has been proposed as a way to maintain and even increase social interaction for pwMS [4]. Taking into consideration the current COVID-19 pandemic situation, online channels may well be the main source of social interaction for pwMS due to social distancing measures imposed in several countries.

In a previous publication, we found that the most recurrent topics related to MS on Twitter were: condition burden; disease modifying drugs; related chronic conditions; and awareness raising [5]. Studies on Twitter show that tweets about MS treatment are often linked to non-neutral sentiments, hinting at the emotional nature of thoughts regarding

\footnotetext{
${ }^{1}$ Corresponding Author, Guido Giunti, University of Oulu, Pentti Kaiteran katu 1, 90014, Oulu, Finland. E-mail: guido.giunti@oulu.fi.
} 
the condition [6]. COVID-19 risk estimations for pwMS still remain unknown, but it is believed that MS patients who are on disease modifying therapies might be at a higher risk of becoming infected, or having a poorer outcome due to their immune system status [7]. There is a lack of evidence regarding the discourse on MS and COVID-19 in social media; the topics and even their emotional responses to events may have shifted during the current outbreak.

This work aims to identify, compare and analyze the sentiment polarity of the topics of MS-related discussion in Twitter before and after COVID-19 pandemic declaration.

\section{Methods}

\subsection{Data extraction and pre-processing}

A tweet collection script using the Twitter API was used to build our initial dataset and has been actively collecting tweets matching the query: \#ms OR \#multiplesclerosis OR "multiple sclerosis". The script has been running since the $9^{\text {th }}$ of February of 2019, and the cutoff point for this study was the $13^{\text {th }}$ of May of 2020. The World Health Organization declared COVID-19 as a pandemic on the $11^{\text {th }}$ of March of 2020 (P-day), which was chosen as the turning point for analyzing the online discourse. An analysis of tweets not related to MS was performed and several terms were identified to filter out and remove tweets not related to MS. The initial dataset was filtered by removing duplicated, re-tweeted, and non-English tweets. A random sample of $n=1000$ tweets was extracted and manually independently binary classified by two authors.

\subsection{Sentiment analysis and data coding}

Sentiment analysis of the pre-processed tweets was performed using SentiStrength [8]. This software is specially designed to analyze the sentiment in short texts by assigning two scores: the intensity of the positive sentiment ( 1 to 5 ) and negative sentiment ( -1 to -5). Each tweet was assigned a positive and negative sentiment score and grouped based on the sentiment categorization. The categorization has been made in four sets: most negative tweets before P-day, most positive tweets before P-day, most negative tweets after P-day and most positive tweets after P-day.

The relevance for each word in our dataset was computed using their frequencyinverse document frequency (tf-idf), which weighs down term frequency with the overall term frequency and reduces the importance of very common terms. The 100 most relevant words used were chosen based on their tf-idf values for deeper analysis.

Building on our previous work [5], we expanded the classification scheme to include topics based on emerging terms from the dataset. Categories were defined as follows: Awareness raising (campaigns for spreading knowledge, mobilize communities, or gather funds for MS); Chronic conditions (other health conditions and diseases of the central nervous system); Condition burden (impact in pwMS); COVID-19: (terms related to the outbreak); Emotional aspects (expression of emotions, family and social support); Healthcare professionals (comments about the healthcare team); MS treatment (drugs, therapies, interventions); and Others.

The 100 most relevant words used in all tweets for each set were manually classified by two authors independently. All discrepancies were solved by consensus. We defined a metric related to the relevance of a category (group of words) as the sum of each word's 
it-idf values of included in this category. We obtained the relevance of each category using this metric for each set. We compared the estimated relevance for each category before and after the cutoff to show changes in the topics of conversation.

\subsection{Ethics approval and funding}

The data protection officer at the University Hospital of North Norway approved this study (Ref:02275). The present work funded by the Business Finland More Stamina project, and the MSF-PHIA project, funded by FEDER (US-1263715).

\section{Results}

An initial dataset with over $610 \mathrm{k}$ tweets was filtered resulting in 147,963 tweets. Manual binary classification showed only a $0.4 \%$ discrepancy in agreements $(n=1000)$. In Figure 1 a summary with the number of words, sentiment before and after P-day per category.

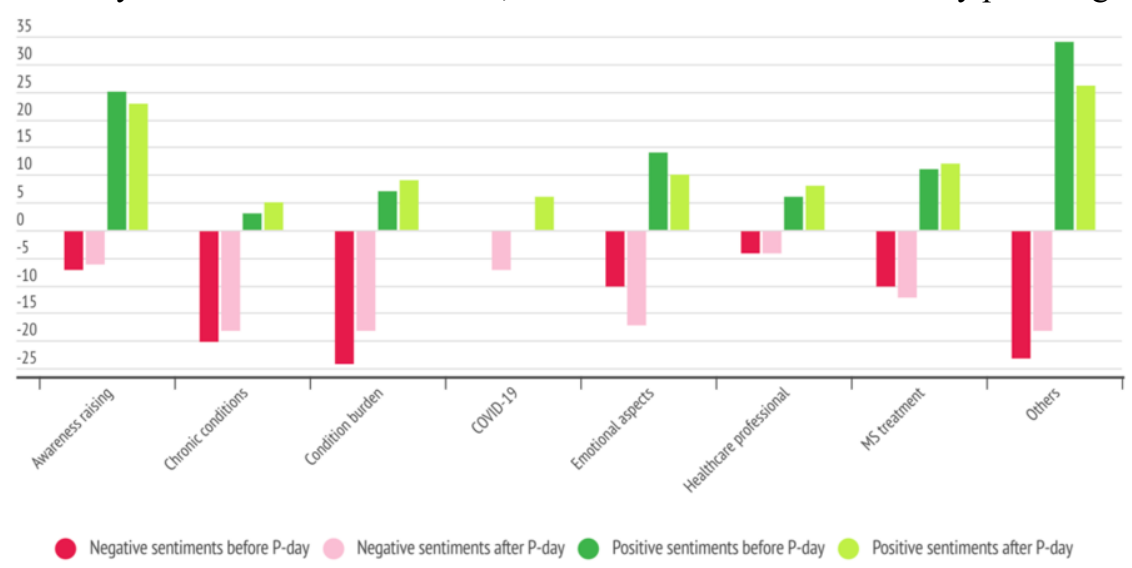

Figure 1. Relevant words with negative and positive sentiment before and after P-day per category.

Categories with more relevant words in tweets with positive sentiment polarity before and after P-day were "Others" (before: 34, after: 26) and "Awareness raising" (before: 25, after: 23). These categories along with "Emotional aspects" reduced in frequency after P-day. All other categories increased slightly after P-day. The "Awareness raising", "Others", "Emotional aspects", and "MS treatment" categories presented the highest estimated relevance values both before and after P-day. There is a reduction in relevance for all of them after P-day. "Healthcare professional", "Chronic conditions", "Condition burden", and "COVID-19" gained positive values. See Table 1. Table 1. Categories with positive tweets with more gain before and after P-day.

\begin{tabular}{ccc}
\hline Categories & Relevance before P-day & Relevance after P-day \\
\hline Awareness raising & $8.22 * 10-3$ & $6.50 * 10-3$ \\
\hline Chronic conditions & $0.80 * 10-3$ & $0.88 * 10-3$ \\
\hline Condition burden & $1.77 * 10-3$ & $2.10 * 10-3$ \\
\hline COVID-19 & $0.00 * 10-3$ & $2.76 * 10-3$ \\
\hline Emotional aspects & $4.42 * 10-3$ & $3.67 * 10-3$ \\
\hline Healthcare professional & $1.84 * 10-3$ & $2.00 * 10-3$ \\
\hline MS treatment & $2.99 * 10-3$ & $2.95 * 10-3$ \\
\hline Others & $7.53 * 10-3$ & $5.44 * 10-3$
\end{tabular}


Categories with more relevant words in negative sentiment polarity tweets before and after P-day were "Condition burden" (before: 24, after: 19), "Others" (before: 23, after: 16), and "Chronic conditions" (before: 20, after: 18). However, there was a noticeable reduction in their frequency after P-day. Messages on "Emotional aspects" showed a significant increment after P-day as "COVID-19" emerged. See Table 2.

Table 2. Categories with negative tweets with more gain before and after P-day.

\begin{tabular}{ccc}
\hline Categories & Relevance before P-day & Relevance after P-day \\
\hline Awareness raising & $1.52 * 10-3$ & $1.21 * 10-3$ \\
\hline Chronic conditions & $5.51 * 10-3$ & $5.25 * 10-3$ \\
\hline Condition burden & $7.83 * 10-3$ & $6.47 * 10-3$ \\
\hline COVID-19 & $0.00 * 10-3$ & $2.10 * 10-3$ \\
\hline Emotional aspects & $2.07 * 10-3$ & $2.99 * 10-3$ \\
\hline Healthcare professional & $2.03 * 10-3$ & $1.05 * 10-3$ \\
\hline MS treatment & $2.63 * 10-3$ & $2.60 * 10-3$ \\
\hline Others & $4.60 * 10-3$ & $3.21 * 10-3$
\end{tabular}

\section{Discussion}

The present study is among the first to cover the way in which people with chronic conditions are reacting to a global pandemic such as COVID-19. Social media can play an important role in understanding attitudes and sentiments related to epidemics [9].

Previous studies have found that most of the topics related to COVID-19 were linked to positive sentiment, and only topics that dealt with deaths and racism were linked with a negative sentiment [9]. However, other studies have reported increases in negative emotions linked to COVID-19, such as anxiety, depression, outrage, and a decrease in positive emotions [10]. A study showed how users shifted from fear to anger, and from sadness to joy in just a few weeks during COVID-19 [11]. In our study, we saw how the conversations around MS on Twitter shifted, displaying both mean negative sentiment increase after COVID-19 was declared a pandemic, and how the relevance of some topics changed. There was a distinct change in regards to MS awareness related tweets, with a decrease in negative tweets and also in positive tweets. This is relevant, as in a previous study it was found that raising awareness was one of the topics linked most to higher positive sentiment [5]. Emotional aspects and the treatments of MS were topics that became less relevant in positive tweets, and also more relevant in negative tweets, therefore these topics probably were experienced in a more pessimistic manner by pwMS.

Emotions affect decision-making [12], a reduction of the negative sentiment of MS related tweets could potentially be beneficial for this population. Public health authorities, healthcare professionals, patient organizations and other stakeholders could help people affected with MS to minimize their negative sentiments by being more present on social media, providing social support and accurate information [13]. Increasing the frequency of emoji use could also have a beneficial effect, as social media posts with emoji are linked to more positive sentiment strength [14].

\subsection{Limitations}

The collected tweets are only those that were publicly available, so it is possible that relevant tweets from pwMS were not captured. Focusing on English speaking tweets also produces a bias in the collection and analysis since there could be conversations in other languages that we are missing. The sentiment analysis was performed with SentiStrength, 
a tool that uses a lexicon-based approach. While there are other sentiment analysis approaches based on machine learning that may perform better [15], SentiStrength was developed using posts on Myspace with good performance on informal text like Twitter.

\section{Conclusions}

Our work provides insight as to how the online conversation of vulnerable populations such as pwMS have changed in view of the COVID-19 pandemic. Understanding how such patient groups feel and react to global health crisis such as the current one can prove useful for designing and developing smart and targeted strategies that could reach them. The use of social media as a way to gauge sentiments towards specific topics is a growing field with potential applications for public health and intervention campaigns.

\section{References}

[1] Browne P, Chandraratna D, Angood C, Tremlett H, Baker C, Taylor B V., et al. Atlas of Multiple Sclerosis 2013: A growing global problem with widespread inequity. Neurology. 2014;83(11):1022-4.

[2] Giunti G, Kool J, Rivera Romero O, Dorronzoro Zubiete E. Exploring the Specific Needs of Persons with Multiple Sclerosis for mHealth Solutions for Physical Activity: Mixed-Methods Study. JMIR mHealth uHealth. 2018 Feb 9;6(2):e37.

[3] Koutsogeorgou E, Chiesi AM, Leonardi M. Social capital components and social support of persons with multiple sclerosis: a systematic review of the literature from 2000 to 2018. Disabil Rehabil. 2020 Nov 19;42(24):3437-49.

[4] Lavorgna L, Russo A, De Stefano M, Lanzillo R, Esposito S, Moshtari F, et al. Health-Related Coping and Social Interaction in People with Multiple Sclerosis Supported by a Social Network: Pilot Study With a New Methodological Approach. Interact J Med Res. 2017 Jul 14;6(2):e10.

[5] Giunti G, Claes M, Dorronzoro Zubiete E, Rivera-Romero O, Gabarron E. Analysing Sentiment and Topics Related to Multiple Sclerosis on Twitter. Stud Health Technol Inform. 2020 Jun 16;270:911-5.

[6] Ramagopalan S, Wasiak R, Cox AP. Using Twitter to investigate opinions about multiple sclerosis treatments: a descriptive, exploratory study. F1000Research. 2014;216:1-9.

[7] Willis MD, Robertson NP. Multiple sclerosis and the risk of infection: considerations in the threat of the novel coronavirus, COVID-19/SARS-CoV-2. J Neurol. 2020 May 17;267(5):1567-9.

[8] Thelwall M, Buckley K, Paltoglou G. Sentiment in Twitter events. J Am Soc Inf Sci Technol. 2011 Feb;62(2):406-18.

[9] Abd-Alrazaq A, Alhuwail D, Househ M, Hamdi M, Shah Z. Top Concerns of Tweeters During the COVID-19 Pandemic: Infoveillance Study. J Med Internet Res. 2020 Apr 21;22(4):e19016.

[10] Li S, Wang Y, Xue J, Zhao N, Zhu T. The Impact of COVID-19 Epidemic Declaration on Psychological Consequences: A Study on Active Weibo Users. Int J Environ Res Public Health. 2020 Mar 19;17(6):2032.

[11] Lwin MO, Lu J, Sheldenkar A, Schulz PJ, Shin W, Gupta R, et al. Global Sentiments Surrounding the COVID-19 Pandemic on Twitter: Analysis of Twitter Trends. JMIR Public Heal Surveill. 2020 May 22;6(2):e19447.

[12] Rand DG, Kraft-Todd G, Gruber J. The Collective Benefits of Feeling Good and Letting Go: Positive Emotion and (dis)Inhibition Interact to Predict Cooperative Behavior. Perc M, editor. PLoS One. 2015 Jan 27;10(1):e0117426.

[13] Zunic A, Corcoran P, Spasic I. Sentiment Analysis in Health and Well-Being: Systematic Review. JMIR Med Informatics. 2020 Jan 28;8(1):e1602.

[14] Gabarron E, Dorronzoro E, Rivera-Romero O, Wynn R. Diabetes on Twitter: A Sentiment Analysis. J Diabetes Sci Technol. 2019 May 19;13(3):439-44.

[15] Siebert C, Hartmann J, Heitmann M, Schamp C. Accuracy of Automated Sentiment Analysis. SSRN Electron J. 2019. 